\title{
LOS PACIENTES DEL MANICOMIO LA CASTAÑEDA Y SUS DIAGNÓSTICOS. UNA PROPUESTA DESDE LA HISTORIA CUANTITATIVA (MÉXICO, 1910-1968) ${ }^{1}$
}

\author{
Andrés Ríos Molina \\ Instituto de Investigaciones Históricas, Universidad Nacional Autónoma de México \\ andresriosmolina@gmail.com \\ Cristina Sacristán \\ Instituto de Investigaciones Dr. José María Luis Mora \\ csacristan@mora.edu.mx \\ Teresa Ordorika Sacristán \\ Centro de Investigaciones Interdisciplinarias en Ciencias y Humanidades, Universidad Nacional Autónoma de México \\ tordorika@yahoo.com \\ Ximena López Carrillo \\ Facultad de Filosofía y Letras, Universidad Nacional Autónoma de México \\ ximenalopezcarrillo@gmail.com
}

Recibido: 18 diciembre 2014; Aceptado: 22 octubre 2015.

Cómo citar este artículo/Citation: Ríos Molina, Andrés; Cristina Sacristán; Teresa Ordorika Sacristán y Ximena López Carrillo (2016), "Los pacientes del Manicomio La Castañeda y sus diagnósticos. Una propuesta desde la historia cuantitativa (México, 1910-1968)", Asclepio 68 (1): p136. doi: http://dx.doi.org/10.3989/asclepio.2016.15

RESUMEN: El Manicomio General La Castañeda, fundado en la Ciudad de México, albergó a 61.480 pacientes entre 1910 y 1968 . El objetivo de este artículo es presentar un panorama general de la población que ingresó a esta institución y los diagnósticos que recibieron los internos, análisis realizado a partir de una base de datos construida con una muestra de $20 \%$ de la población total. El artículo se divide en tres partes: en la primera, proponemos tres etapas para comprender la historia de La Castañeda cuya periodización es definida por cambios demográficos que coinciden con reformas administrativas; en la segunda, exponemos las características generales de la población psiquiátrica de La Castañeda; y finalmente, describimos los principales cambios demográficos, los cuales obedecieron a factores socio-políticos, innovaciones tecnológicas y transformaciones en la clínica. Como características principales de la población de La Castañeda tenemos la brevedad del encierro (18 meses en promedio) y la reducida mortalidad (23.8\%) en comparación con otras instituciones del mismo tipo. En dicha circulación poblacional encontramos que las familias desempeñaron un papel determinante al asumir el cuidado de sus parientes locos. Así, el encierro prolongado de pacientes crónicos no fue un problema que aquejara al Manicomio General de forma tan severa como se ha creído.

PALABRAS CLAVE: La Castañeda; Población Psiquiátrica; Enfermedades Mentales; Manicomio; México.

THE PATIENTS AND THEIR DIAGNOSIS AT LA CASTAÑEDA GENERAL ASYLUM. AN APPROACH FROM QUANTITATIVE HISTORY (MEXICO, 1910-1968)

ABSTRACT: During its 58 years in operation (1910-1968), the Manicomio General La Castañeda housed 61,480 people. In this paper, we present an overview of the general characteristic of the patients based on a $20 \%$ sample of the overall population. We divided the text in three sections: in the first part we argue that the history of the institution comprises three distinctive periods characterized by demographic changes that coincide with administrative reforms. In the second, we present the general characteristics of La Castañeda's psychiatric population. Finally, we describe the most salient demographic changes, which stemmed either from socio-political events, technological innovations or clinical transformations. Some of the most salient results of the analysis of the sample show that the inmate population had short periods of hospitalization in the asylum (an average of 18 month), as well as a lower mortality rate (24.2\%) in comparison to contemporary mental institutions. Families played a fundamental role in the care of their mad relatives, which accounts for the relatively short periods of hospitalization as well as the low death rates. Consequently, for this particular institution, chronic patients weren't such a serious problem as believed.

KEY WORDS: La Castañeda; Psychiatric Population; Mental Illness; Mental Asylum; Mexico.

Copyright: (c) 2016 CSIC. Este es un artículo de acceso abierto distribuido bajo los términos de la licencia Creative Commons Attribution (CC BY) España 3.0. 


\section{INTRODUCCIÓN}

En 1910 abrió sus puertas el Manicomio General La Castañeda, institución que durante 58 años se ocupó del cuidado y tratamiento de los enfermos mentales en México. Con ello, reemplazó a los hospitales para dementes que habían sido fundados en la Ciudad de México en los tempranos años de la época colonial. De esta manera, el Manicomio formó parte del programa de modernización urbana impulsado por el gobierno de Porfirio Díaz desde fines del siglo XIX en el terreno de la salud. Dicha reforma sanitaria condujo a la construcción de hospitales, campañas de prevención y vacunación, creación de una policía sanitaria, la aprobación del primer código sanitario, por mencionar sólo algunas acciones (Agostoni, 2003; Carrillo, 2010). La construcción de la nueva institución psiquiátrica inició en 1908, cubría una extensión de $141.662 \mathrm{~m}^{2}$ en los terrenos de la antigua Hacienda La Castañeda, y fue planeada para albergar 1300 pacientes.

El nuevo Manicomio fue considerado por las élites como un incuestionable signo de modernización en materia de atención psiquiátrica, que garantizaba un lugar para México a la «altura de los países avanzados» (Ríos, 2009a). Además de haber atendido a 61.480 pacientes, fue un espacio central para la formación de psiquiatras, neurólogos, enfermeras, psicólogos, psicómetras y trabajadoras sociales. Sin embargo, pasado el tiempo resultó insuficiente: a fines de los años cuarenta llegó a albergar hasta 3.800 pacientes y las condiciones de insalubridad y el hacinamiento se hicieron más evidentes, por lo que se creó una leyenda negra que se agudizaba con artículos periodísticos donde se exhibía a esta institución en decadencia total. Finalmente, el Manicomio La Castañeda fue clausurado en 1968 tras casi seis décadas de existencia (Vicencio, 2014).

Esta institución psiquiátrica ha sido analizada por la historiografía mexicana, particularmente desde dos perspectivas: la dinámica administrativa (Sacristán, 2001, 2002, 2003, 2005a, 2005b, 2008, 2010a, 2010b; Vicencio, 2014) y las narrativas de los pacientes a partir de los expedientes clínicos (Rivera 2001a, 2001b, 2001c; Mancilla, 2001; Ríos, 2004, 2009a, 2009b). Sin embargo, la historiografía sobre La Castañeda carece de una mirada global sobre los pacientes, ya que la mayoría de los estudios realizados se han concentrado en casos particulares y en los primeros años de funcionamiento de la institución. Por consiguiente, la propuesta de este artículo es presentar un panorama general de la población que ingresó en La Castañeda y los diagnósticos que recibieron a lo largo de cincuenta y ocho años a partir de la metodología de la historia cuantitativa.
Para abordar la mencionada población psiquiátrica hemos construido una base de datos a partir de una muestra aleatoria de $20 \%$ del total de la población, es decir, 12.296 registros. De casi todos ellos se recuperó la información correspondiente a once variables que a lo largo de toda la historia del Manicomio se asentaron en los libros de registro: sexo, edad, lugar de nacimiento, lugar de residencia, ocupación, estado civil, instancia remitente, enfermedad diagnosticada, tiempo de estancia, condición de salida (muerto, curación/ remisión/apto para vivir en familia, fuga o por solicitud de la familia) y número de reingresos. Para efectos de clasificación, de la base de datos excluimos 257 registros sin diagnóstico, 1,361 casos que recibieron más de un diagnóstico al momento del ingreso, y 42 casos con enfermedades no psiquiátricas. Por consiguiente, en este artículo trabajamos con los restantes 10.641 registros que recibieron un solo diagnóstico al momento del ingreso, es decir, $86.5 \%$ de la muestra.

Una vez construida la base de datos tomamos la variable "diagnóstico», asentada en los libros de registro, para determinar si era posible descubrir algún sistema clasificatorio utilizado en el Manicomio. La base arrojó un total de 2.150 maneras diferentes de nombrar la enfermedad mental, confirmando lo indicado por Rafael Huertas:

Cuanto mayor sea el número de historias estudiadas, mayor será la variedad terminológica utilizada, pudiendo llegar a constituir un amplísimo abanico de posibilidades diagnósticas -y terapéutica- que es preciso jerarquizar (Huertas, 2012, p. 115).

¿Cómo clasificar y hacer manejables estas 2.150 posibilidades que teníamos ante nosotros? Este mismo problema lo enfrentó Ana Conseglieri quien analizó los diagnósticos de los pacientes que ingresaron al Hospital de Leganés en España durante la posguerra, en cuyos libros de registro encontró 140 diagnósticos para tan solo 615 pacientes; diagnósticos que fueron clasificados según el modelo de Kraepelin, el cual fue ampliamente utilizado, tal y como refieren diversas fuentes consultadas (Conseglieri, 2014, p. 136).

En nuestro caso, no encontramos cuadros, tablas o algún tipo de formato estadístico para clasificar los diagnósticos, ni documento alguno que nos permitiera intuir alguna forma de estandarizar los criterios diagnósticos usados en La Castañeda; tópico también ausente en los artículos publicados o las tesis presentadas en la época. De manera excepcional contamos con dos documentos que nos permiten ver intentos por clasificar a los pacientes, pero que no obedecieron a políticas ni a criterios institucionales: el primero 
es un estudio del médico Enrique Aragón hecho en 1925 en La Castañeda, donde los criterios son confusos $^{2}$; y el segundo es la Primera Investigación Nacional de Enfermos Neurológicos y Psiquiátricos ${ }^{3}$, censo levantado a partir de una encuesta nacional aplicada a médicos e instituciones en 1960 y publicada en 1964, siguiendo los criterios del CIE.

En un principio esta ausencia nos llevó a pensar que no hubo un sistema compartido por los psiquiatras del Manicomio General para clasificar a sus pacientes. Sin embargo, una cita encontrada en el expediente clínico de un paciente esquizofrénico que ingresó en 1911 nos llevó a replantear nuestra postura:

En efecto, es sabido que se considera como predominante en el cuadro de dicha afectividad [la esquizofrenia] la disminución afectiva y Kraepelin dice que es una afección ante todo emocional [...] Entre las especies de demencia precoz [la del paciente] no cabe en la forma paranoide, por no ser el delirio predominante ni sistematizado; ni en la catatónica por no ser culminantes los movimientos estereotipados, sino hebefrénica, desarrollada previamente en la juventud y llevando rápidamente a la demencia [...] en algunos casos hay remisiones, en las cuales los enfermos, conservando algo de su capacidad intelectual, pueden dedicarse al trabajo, aunque en menor escala; así el artista se vuelve artesano, el jurista notable ayudante de abogado, el mecánico en operario; pero en el $75 \%$ de los casos, la terminación es la demencia completa ${ }^{4}$.

Es clara la influencia de Kraepelin en la definición de esquizofrenia, en las características de los tres tipos (hebefrénica, catatónica y paranoide) y en la convicción de la demencia como inevitable destino. Siguiendo esta idea, poco a poco se hizo evidente que el modelo de Kraepelin había sido utilizado en La Castañeda para la clasificación de los pacientes en la práctica clínica. Por consiguiente, al igual que en la mencionada investigación sobre el Hospital de Leganés, encontramos coherentes los diagnósticos del Manicomio General con los catorce grupos propuestos por Kraepelin en la sexta edición de su Tratado publicado en 1899, como se observa en la tabla anexa al final de este artículo.

Una vez clasificados los diagnósticos definimos, en primer lugar, las características de la población global de La Castañeda y, en segundo lugar, las particularidades según los grupos de diagnósticos. Dicha revisión nos señaló que los cambios en la población obedecieron a fenómenos tanto externos como internos al campo psiquiátrico. Debido a la cantidad de información recogida en la base de datos, en este artículo desarrollaremos, a partir de cinco ejemplos muy concretos que involucran al $70.9 \%$ de la población de La Castañe- da, la forma en que los contextos socio-político, tecnológico y clínico fueron determinantes en los aumentos y disminuciones de las afecciones más diagnosticadas. Por ejemplo, la influencia de fenómenos político-sociales se hizo evidente en los pacientes con alcoholismo, cuya reducción a partir de 1929 coincidió con la Campaña Nacional Antialcohólica impulsada por diferentes instancias del gobierno con propaganda masiva, eventos populares, desfiles, programas radiales, etc. A su vez, el aumento de ingresos por toxicomanía coincidió con la aparición, en 1931, del Reglamento contra las Toxicomanías, a través del cual se patologizaba el consumo de drogas y se estipulaba que las autoridades estaban en la obligación de llevar a estos «enfermos» al Manicomio General; tal crecimiento se acentuó en 1933 con la creación del Hospital Federal de Toxicómanos. Así, estas medidas de control social aplicadas por las instancias de poder aparecen como determinantes en la dinámica de la población diagnosticada con alcoholismo y toxicomanía.

En cuanto a los cambios tecnológicos, la incorporación del electroencefalógrafo en 1934 trajo consigo un aumento en la población diagnosticada con epilepsia, ya que el nuevo aparato podía detectar un foco epiléptico que permitía relacionar un cuadro clínico con una lesión cerebral. En el mismo tenor de los cambios generados por la tecnología, el uso de la penicilina a mediados de siglo XX trajo consigo una reducción sustancial en la cantidad de pacientes diagnosticados con parálisis general progresiva.

Finalmente, encontramos cambios que tuvieron lugar en el terreno de la clínica: la recepción de Kraepelin en La Castañeda se evidencia en el uso de las categorías propuestas por este reconocido psiquiatra en el diagnóstico de las llamadas "oligofrenias» y las "psicosis endógenas», particularmente la demencia precoz y la psicosis maniaco-depresiva. La primera, que para la década de 1920 sería remplazada por el término esquizofrenia, fue la enfermedad más diagnosticada en el Manicomio General, $19.9 \%$ de la totalidad de pacientes de la muestra recibió dicho diagnóstico. Este uso revela un progresivo abandono de los criterios clasificatorios franceses que habían sido utilizados desde finales del siglo XIX; proceso posibilitado por la aparición de la primera generación de psiquiatras jóvenes que le dieron la bienvenida a Kraepelin en mayo de 1925 cuando visitó el Manicomio La Castañeda $a^{5}$. Antes de describir cada uno de los cambios mencionados, expondremos las características principales del Manicomio a lo largo de sus cincuenta y ocho años de vida y la dinámica general de su población. 


\section{EL MANICOMIO GENERAL LA CASTAÑEDA: TRES MOMENTOS}

Para el abordaje histórico de esta institución proponemos una periodización que obedece a los cambios observados en el número de ingresos anuales (gráfica 1), y que además, coincide con la propuesta de Cristina Sacristán a partir de fuentes administrativas: un primer momento en que decrece la población psiquiátrica, el cual concuerda con la Revolución mexicana (1910-1919); un segundo periodo conocido como la posrevolución (1920-1944) en el cual aumentó la cantidad de ingresos por año pasando de 684 en 1920 a 1.663 en 1944; y un tercer momento (1944-1968) donde se estabilizó el número de ingresos e inició un proceso de descentralización, posibilitado por la apertura de nuevas instituciones psiquiátricas destinadas a pacientes remitidos desde La Castañeda en aras de atacar el sobrecupo que venía padeciendo tiempo atrás, lo que finalmente contribuyó a su clausura. Veamos cada uno de estos momentos.

\section{Decrecimiento, 1910-1919}

Inaugurado el Manicomio en 1910, estalló la Revolución y llegó, de manera paralela, la inestabilidad política, económica y administrativa. Si bien no encontramos reportes de una notable crisis o desabasto distinta al resto de las que habría después (Ríos, 2009), durante los diez años de la etapa armada de la Revolución hubo doce directores titulares y tres interinos, así como una alta movilidad entre los médicos, ya que de los 98 contratados en los primeros diez años, 82 ya no laboraban en 1921 (Sacristán, 2010a). Lo más llamativo, sin embargo, es la reducción en la cantidad de ingresos por año que se puede apreciar en la gráfica 1. Dichos ingresos disminuyeron hasta alcanzar un punto realmente bajo en 1915, justamente cuando la guerra civil estaba en su momento más álgido debido a la cantidad de epidemias, falta de agua y de comida que, con razón, hicieron que aquel año fuera conocido como el «año del hambre» (Rodríguez, 2010). Como suele ocurrir en contextos de guerra, los parámetros para definir lo normal y lo anormal se diluyeron, repercutiendo en un menor número de internos en los manicomios (Vázquez, 2013).

\section{Crecimiento, 1920-1944}

Al iniciar la década de 1920 aparecieron informes sobre el mal estado de las instalaciones del Manicomio, ya que durante la Revolución el mantenimiento fue precario: ausencia de colchones y sábanas, ventanas con los vidrios rotos, plagas de ratas y una farmacia raquítica. En esos años las instalaciones se descompusieron, los enseres se dañaron y no hubo recursos suficientes para cambiarlos (Sacristán, 2010a). En esta década inició el aumento de la población psiquiátrica que se detuvo en 1944, año donde se registra la mayor cantidad de ingresos: 1.663 pacientes. El crecimiento en el número de internos lo podemos comprender desde varias perspectivas, entre ellas, el aumento de la población nacional que pasó de 14.2 millones en 1920 a 35 millones en 1960 (Mendoza, 2010; Mier y Terán, 1991), y las campañas contra la mendicidad con las consecuentes razias

Gráfica 1. Cantidad de pacientes que ingresaron al Manicomio La Castañeda, 1910-1968.

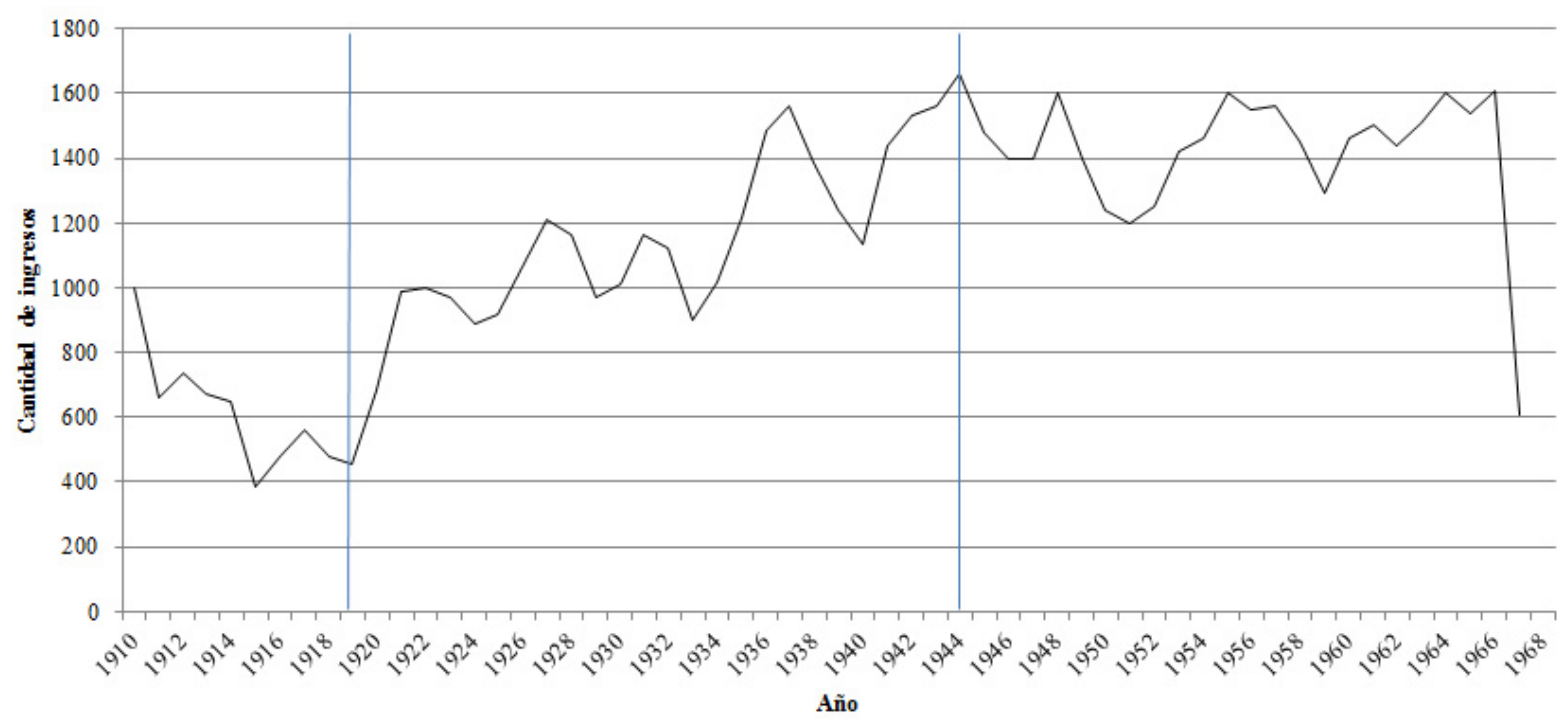


impulsadas por el gobierno capitalino. Los camiones de la Beneficencia Pública recogían en las calles a todos los que no pudieran demostrar un domicilio fijo. Una vez en las oficinas, éstos eran remitidos a las diferentes instituciones asistenciales, entre ellas, La Castañeda. Sin embargo, solían ser indigentes con altos grados de desnutrición, deshidratación y alcoholismo, quienes al cabo de unos cuantos meses eran dados de alta (Ríos, 2010). Es probable que la presencia de pacientes sin claros síntomas psiquiátricos haya alentado el otorgamiento de permisos de salida, pues llama la atención que entre 1930 y 1950 una cantidad notable de pacientes no retornaron tras el permiso que se les otorgó (gráfica 2).

Este crecimiento de la población psiquiátrica también estuvo aparejado a la profesionalización de la psiquiatría. A diferencia de los años revolucionarios donde imperó la inestabilidad institucional, a partir de 1929 el Manicomio comenzó a ser dirigido por una nueva generación de psiquiatras que profesionalizó la psiquiatría en México; entre ellos destacan Alfonso Millán, Edmundo Buentello, Samuel Ramírez Moreno, Manuel Guevara Oropesa y Mathilde Rodríguez Cabo. Según Héctor Pérez-Rincón:

fueron ellos quienes iniciaron la asistencia propiamente médica de los enfermos mentales en los escasos centros asistenciales públicos y privados dedicados a su cuidado, y establecieron la enseñanza formal de la neurología y la psiquiatría (Pérez-Rincón, 1995, p. 45).
Dichos psiquiatras fueron los que regularizaron los procesos de ingreso, promovieron una Ley General de Alienados «que respaldara la acción de la psiquiatría frente a otros poderes y protegiera al enfermo de múltiples abusos», e impulsaron la reorganización del Manicomio. Como parte de esta reorganización se abrió un consultorio y un laboratorio para análisis clínicos, se creó un pabellón de toxicómanos, uno de psiquiatría infantil, una escuela de niños anormales, se renovaron espacios que requerían mantenimiento y se implementaron nuevas prácticas terapéuticas como las actividades deportivas. Finalmente, y como muestra de la profesionalización que tenía lugar, comenzaron a circular dos revistas especializadas: Revista Mexicana de Psiquiatría, Neurología y Medicina Legal (1934) y Archivos de Neurología y Psiquiatría de México (1937) (Sacristán, 2010a).

\section{Estabilización, 1944-1968}

A partir de 1944 comenzó el proceso de descentralización de la atención psiquiátrica y La Castañeda dejó de ser la institución que inexorablemente recibía pacientes de todo el país. La Operación Castañeda fue una estrategia para crear una red de hospitales psiquiátricos en diferentes estados de la república, para poco a poco reducir la población del Manicomio General y finalmente clausurarlo en 1968. Después de numerosas solicitudes por parte de los directivos, donde señalaban la cantidad de problemas que gene-

Gráfica 2. Condición de salida por año, 1910-1968 (\%)

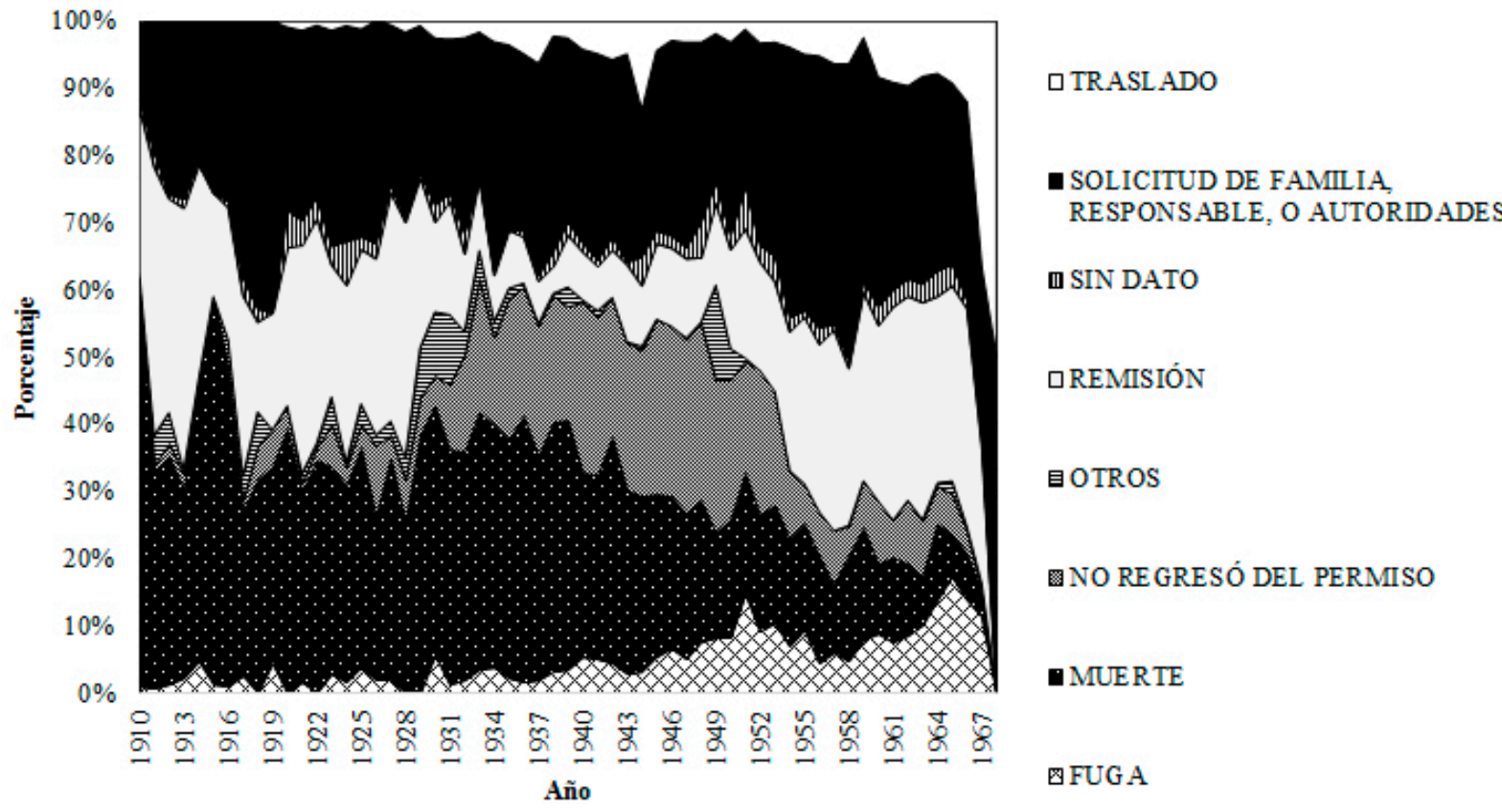


raba la saturación de pacientes, en 1945 fue inaugurada la primera granja para enfermos mentales localizada en San Pedro del Monte (Guanajuato) en una extensión de 92 hectáreas con capacidad para 400 enfermos. Esta propuesta buscaba desahogar el Manicomio mediante el traslado de los pacientes crónicos considerados incurables, y permitir que los agudos recibieran los beneficios de la ciencia (Sacristán, 2013). Esta granja fue la primera de doce instituciones erigidas para remplazar a La Castañeda: seis hospitalesgranja, tres hospitales campestres, un albergue y dos hospitales psiquiátricos para agudos (Vicencio, 2014). Además, en 1964 se inauguró el Instituto Nacional de Neurología y Neurocirugía y diversas instituciones psiquiátricas en Jalisco, Monterrey y Yucatán. La creación de estas instituciones nos permite comprender el estancamiento en el crecimiento de la población de La Castañeda, el cual se mantuvo en un promedio de 1,500 ingresos por año entre 1944 y 1968.

\section{CARACTERÍSTICAS DE LA POBLACIÓN PSIQUIÁTRICA DE LA CASTAÑEDA}

La población de la capital mexicana se quintuplicó entre 1910 y 1960: pasó de 720.753 a 4.870 .876 habitantes, en un país que en el mismo lapso de tiempo duplicó su población, de 15.160 .369 a 34.923.128. Por una parte, el crecimiento demográfico que tuvo lugar entre 1919 y 1940 fue congruente con el aumento de la población nacional. No obstante, entre 1940 y 1950 la población de la capital se duplicó (de 1.757 .530 a 3.050.442), mientras que la cantidad de ingresos pasó de 1.055 en 1940 a 1.410 en 1950. Debido a que carecemos de información sobre las instituciones psiquiátricas privadas y sobre pabellones psiquiátricos construidos en diferentes hospitales de la república, no es posible tener información global que nos permita comparar la cobertura ofrecida por La Castañeda frente a otras instituciones. Sin embargo, es un hecho que después de 1920 hubo un marcado problema de sobrepoblación: con capacidad para 1.300 internos originalmente, albergó hasta 3.500 a finales de la década de 1930. Población numerosa si la comparamos con el Hospital Leganés en España o las instituciones británicas a fines del siglo XIX, las cuales no alcanzaban los 600 internos (Renvoize y Beveridge, 1989). Sin embargo, tampoco consiguió las proporciones colosales de la Salpêtrière en los tiempos de Charcot, ni del Hospicio Oliva en Córdoba (Argentina) que en la década de 1930 albergó 4 mil pacientes (Ablard, 2008, p. 75). Así, pese al aumento de la población tanto nacional como capitalina, el aumento en la cantidad de internamientos no fue proporcional debido a la crea- ción de nuevas instituciones que fueron desplazando a La Castañeda como el único espacio psiquiátrico del país. Veamos las principales características de los habitantes de La Castañeda.

La edad promedio de los pacientes de la institución fue de 33.6 años. La población más joven la encontramos en el grupo diagnosticado con retraso mental, con 20.2 años en promedio, mientras que los de mayor edad fueron diagnosticados como seniles o pre-seniles con 64.2 años. Cabe mencionar que otra enfermedad que supera la tendencia general es la parálisis general progresiva cuya población tenía una edad promedio de 39.6 años.

Con relación al estado civil tenemos la siguiente distribución: solteros, 54.6\%; casados, $29.6 \%$; viudos, $5.9 \%$; en unión libre o amancebados, $1.2 \%$; divorciados, $0.5 \%$; y sin información, $8.3 \%$. Esta distribución fue constante en los diferentes grupos de diagnósticos, con excepción de los pacientes con parálisis general progresiva, pues $53.2 \%$ de dicha población eran casados que generalmente salían del Manicomio por solicitud de la familia y muchas veces gracias a la gestión hecha por la misma esposa.

En cuanto a la diferencia por sexo, siempre hubo más hombres (64.2\%) que mujeres (35.8\%). ¿Cómo explicar esta diferencia constante en un país con una población con más mujeres que hombres? En este punto en particular nos limitamos a formular hipótesis que podrán ponerse a prueba con el análisis de los expedientes clínicos posteriormente. Por una parte, podríamos entender esta superioridad numérica por el importante número de internos que fueron remitidos por instituciones de control social, como los cuerpos de seguridad, estableciéndose una clara relación entre trasgresión del orden público y enfermedad mental en los hombres (véase tabla 1). Pero por otro lado, también podemos suponer que en una sociedad donde las redes de parentesco han desempeñado un papel fundamental en la estructuración de las relaciones sociales, la mujer estuvo controlada por la familia ya que desempeñaba un papel social propio de ama de casa orientado a las labores domésticas. En consecuencia, era muy probable que las familias se encargaran del cuidado de una enferma mental al mantenerla en el espacio propio para la mujer según los criterios culturales de la época: el doméstico. El único grupo de diagnósticos donde se invirtió la proporción entre sexos fue en los diagnósticos de psicosis maniaco-depresiva (23\% hombres y $77 \%$ mujeres) y la diferencia más notable la encontramos en los pacientes con histeria ( $13 \%$ y $87 \%$ ), quienes pasaron poco tiempo en el encierro psiquiátrico (8 meses). 
Tabla 1. Grupos de instancias remitentes, 1910-1968

\begin{tabular}{|c|c|}
\hline Instancias remitentes & (\%) \\
\hline No se sabe & 35.0 \\
Asistencia & 26.6 \\
Seguridad & 13.5 \\
Estatal & 10.5 \\
Familia & 9.2 \\
Hospitalaria & 4.6 \\
Voluntad propia & 0.4 \\
Otros & 0.2 \\
\hline \multicolumn{2}{c}{ Total } \\
\hline
\end{tabular}

Si cruzamos las variables tiempo de estancia y condición de salida encontramos lo siguiente: los pacientes pasaron un promedio de 18.1 meses encerrados, $24.2 \%$ falleció en el encierro y $29.4 \%$ fueron dados de alta por solicitud de la familia (tabla 2). La tendencia a permanecer internos menos de dos años, aunado a una baja cantidad de muertes en La Castañeda y una alta proporción de salidas por intervención de la familia, nos hace pensar en una institución con una alta circulación poblacional donde, si bien hubo pacientes crónicos que pasaron ahí toda su vida, éstos constituyeron una minoría frente a la multitud de personas que entraban y salían. Por esta razón es necesario pensar a La Castañeda, no sólo cómo un espacio de reclusión perpetua sino como un sitio de paso donde la cronicidad no debió ser el problema más serio de la institución (Ríos, 2009c).

Tabla 2. Grupos de condición de salida, 1910-1968

\begin{tabular}{|c|c|}
\hline Condición de salida & $\%$ \\
Solicitud de responsable o familiar & 29.4 \\
Muerte & 24.2 \\
$\begin{array}{c}\text { Curación/Mejoría/Remisión/Apto para vivir } \\
\text { en familia }\end{array}$ & 19.9 \\
No regresó de permiso & 12.3 \\
Traslado & 4.4 \\
Fuga & 5.7 \\
Otros & 1.9 \\
Sin dato & 2.2 \\
\hline \multicolumn{2}{|c}{ Total } \\
\hline
\end{tabular}

Si observamos la condición de salida en una perspectiva histórica (gráfica 2), veremos que en los primeros años de La Castañeda hubo un alto porcenta- je de pacientes que fallecieron, particularmente en 1915, cantidad que se fue reduciendo hasta llegar a la mitad después de 1944. Además, entre 1930 y 1951 hubo un alto porcentaje de pacientes con ciertos signos de recuperación que recibieron un pase de salida por unos cuantos días después de los cuales «no regresaron de permiso». Desconocemos si lo anterior se relacione con una estrategia deliberada de autorizar la salida temporal para que los pacientes se reincorporaran a la vida social y los familiares se hicieran responsables de ofrecerles los cuidados necesarios. Por otra parte, entre 1952 y 1968 hubo un notable descenso en la mortalidad y un incremento en el número de fugas, lo cual permite suponer una reducción en los mecanismos del control de los internos, máxime considerando que la arquitectura de La Castañeda era poco apropiada para proporcionar una custodia tipo carcelaria. Aunado a lo anterior, hubo un notable incremento en la cantidad de pacientes que salieron «mejorados», "curados» o «en remisión», lo cual nos permite sugerir un aumento en la eficacia terapéutica de la institución ya que fue a partir de dichos años cuando se comenzaron a utilizar tratamientos farmacológicos (antipsicóticos y anticonvulsivos). No obstante, calibrar la eficacia terapéutica de La Castañeda, va más allá de los límites de este artículo.

En cuanto al lugar de origen de los internos, 32.3\% de la población nació en el Distrito Federal, lugar donde se encontraba el Manicomio; 30.6\% procedía de los estados próximos a éste (Guanajuato, Hidalgo, Michoacán, Morelos, Puebla, Querétaro y Tlaxcala), 35\% provenían de estados más alejados (Coahuila, Chihuahua, Sonora, Sinaloa, Chiapas, Oaxaca, Campeche, Yucatán, entre otros), 2.1\% lo comprendían extranjeros, cuyas dos terceras partes habían nacido en España, Estados Unidos, China, Alemania, Cuba e Italia, a los que se sumaban los provenientes de 18 países más de América, Europa y Asia. Por consiguiente, encontramos que $65.6 \%$ de la población de La Castañeda eran migrantes llegados a la capital mexicana. Con relación a la inserción de estos migrantes en la vida laboral, encontramos que $6.7 \%$ tenían como oficio el de «campesino" o "agricultor», 32\% no registra oficio alguno, y el restante $61.3 \%$ tenían trabajos propios de la vida urbana: una larga lista de 708 oficios y profesiones que iba desde abogados, agentes de seguros, ingenieros, filarmónicos, políticos o militares, hasta voceadores, electricistas, estudiantes, choferes, mecánicos, para mencionar sólo unos cuantos, lo que confirma la estrecha relación entre el fenómeno urbano y las instituciones psiquiátricas. Así, nos encontramos con una alta población migrante que formó parte de la vida laboral propia de la capital mexicana. 
Las instancias remitentes que más pacientes enviaron fueron las instituciones de asistencia, entre las que destacan la Beneficencia Pública y la Secretaría de Asistencia, que en 1944 se convirtió en Secretaría de Salubridad y Asistencia, además de asilos, hospicios y casas de ancianos. La cantidad de pacientes remitidos por dichas instancias obedece a que el reglamento estipulaba que quien llegara a las puertas del Manicomio sólo podía ser ingresado si presentaba dos certificados médicos donde se mencionara la perentoriedad del encierro. Cuando las familias no tenían recursos para pagar un médico particular, éstas acudían a la Beneficencia o a la Asistencia para que se les emitieran los certificados de manera gratuita, por lo cual es probable que el porcentaje de pacientes remitidos por la familia sea superior a 9.2\% (tabla 1). Por otra parte, queremos resaltar la cantidad de pacientes que fueron enviados por las instancias de seguridad (13.5\%), principalmente la policía y las cárceles, las escuelas correccionales y el Tribunal para Menores Infractores. Esto quiere decir que La Castañeda, además, fue un espacio asociado al control social.

A manera de síntesis, encontramos como una de las características principales de la población psiquiátrica de La Castañeda las bajas cifras de mortalidad: sólo 24.2\% falleció en el encierro; porcentaje bajo si lo comparamos con el Hospital de Leganés, donde 71\% de los pacientes fallecieron en el encierro durante la Guerra Civil en España (Vázquez, 2013, p. 183) y 59.4\% durante el franquismo (Conseglieri, 2014, p.106). Una segunda característica tiene que ver con la brevedad del periodo de internamiento, 18 meses, si lo comparamos con la mencionada institución española (6.25 años durante la Guerra Civil y 7.78 durante la posguerra) o con las instituciones de la costa este de Estados Unidos donde se alcanzaba hasta 5 años en el encierro (Grob, 1994, pp. 119-123). En tercer lugar, si bien hubo internos remitidos por instancias como la policía o correccionales, la mayoría de los pacientes de La Castañeda era dada de alta ya fuera por remisión, por solicitud de la familia o por fuga, hecho que nos lleva a cuestionar la idea generalizada de encierro perpetuo, y comenzar a verla como un sitio de tránsito en el que estaban internados durante año y medio en promedio, y donde la familia jugó un papel determinante al momento de solicitar el alta y optar por hacerse cargo de sus locos en lugar de dejarlos en el abandono.

\section{EL CONTROL ESTATAL DEL ALCOHOLISMO Y LAS TOXICOMANÍAS}

Tras la inauguración de La Castañeda, el incremento en los ingresos por alcoholismo fue muy notorio, al punto de representar $45 \%$ de la totalidad de los internamientos en 1912, manteniéndose constante hasta 1929 cuando inició un descenso en la cantidad de ingresos (gráfica 3). Esta reducción llegó al punto más bajo en 1933, cuando comenzó lentamente a aumentar hasta alcanzar en 1957 la misma cantidad de in-

Gráfica 3. Ingresos por año de pacientes con alcoholismo y toxicomanía al Manicomio General, La Castañeda, 1910-1968

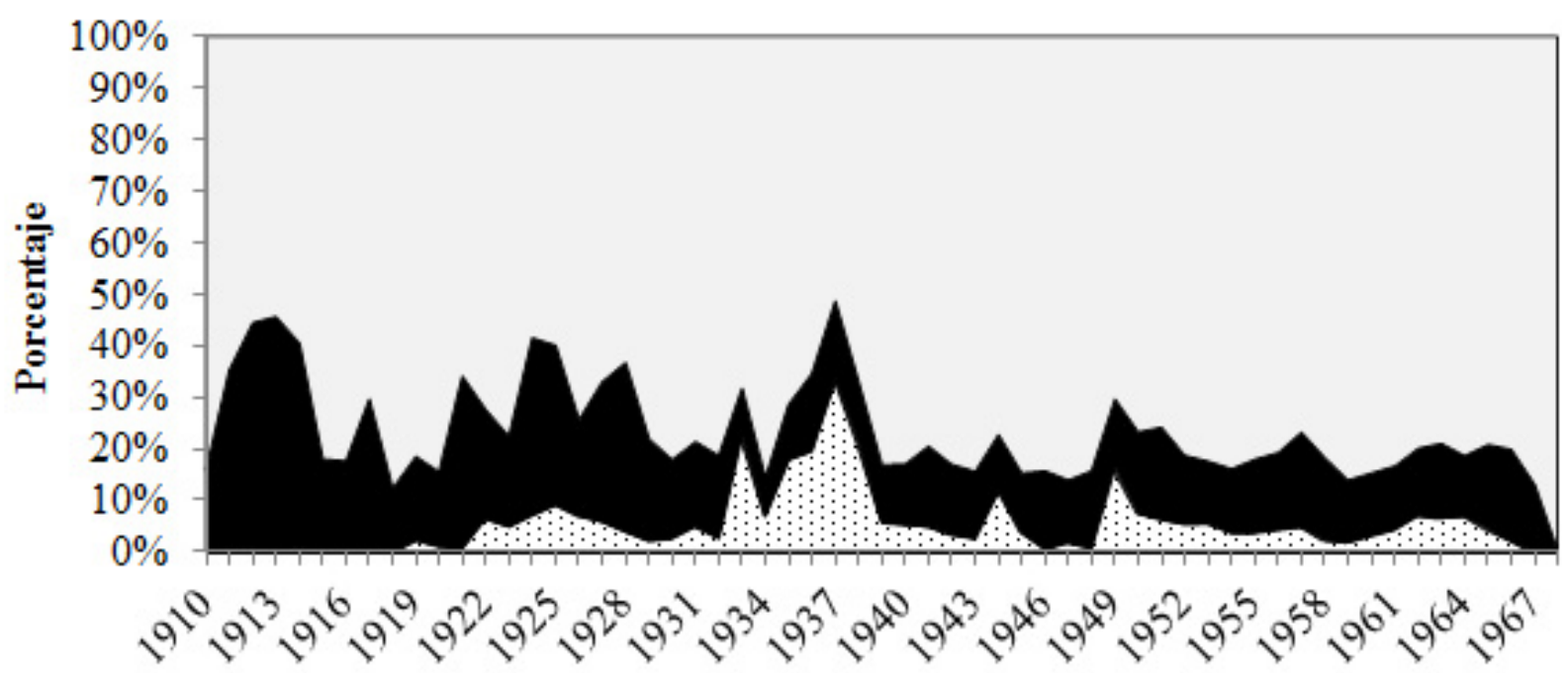

Año

\section{TOXICOMANÍA -ALCOHOLISMO $\square$ POBLACIÓN GENERAL}


gresos que en 1928. El mencionado decrecimiento en la población de alcohólicos puede estar relacionado con las campañas antialcohólicas que tuvieron lugar en el país y particularmente en la Ciudad de México en 1929, año en el que se organizó el Comité Nacional de Lucha contra el Alcoholismo. Bajo la lógica de la "dictadura sanitaria» impulsada desde la Constitución de 1917, el alcohólico fue considerado como un «degenerado" que amenazaba el proyecto de nación, razón por la cual intensas campañas mediáticas fueron consideradas como herramientas para eliminar este hábito. Así, el despliegue de recursos y personas en una labor pedagógica mediante conferencias, teatro guiñol, cine, folletos impresos y uso de la televisión, pudo ser efectivo si consideramos la reducción de ingresos a La Castañeda a partir de la campaña de 1929 (Gudiño, 2009, pp. 162-181; Gudiño, 2008).

Con alguna variedad de alcoholismo fue diagnosticado el $19.3 \%$ del total de internos, quienes alcanzaron una estancia promedio de 8.3 meses, lapso bastante breve en comparación con el promedio general. En relación con el sexo, los datos son muy relevantes: mientras que la población general de la muestra está compuesta por $64.2 \%$ de hombres y $38.8 \%$ de mujeres, la población de alcohólicos es eminentemente masculina: $87.8 \%$ de hombres y $12.2 \%$ de mujeres.

En relación con la instancia remitente, la policía envió al Manicomio a $16 \%$ de los alcohólicos, la Asistencia Pública a 6.8\%, la Beneficencia Pública a 13.1\%, por orden de algún gobernador $6.9 \%$, pero por solicitud de

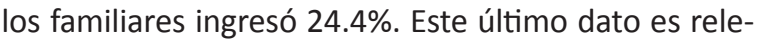
vante ya que el promedio general de pacientes remitidos por la familia para toda la base de datos es de 9.2\%. Ello significa que sobre los alcohólicos hubo un control doméstico significativo traducido en el encierro psiquiátrico, con un matiz de acción disciplinaria.

Otro hábito que fue patologizado y combatido por el Estado fue la toxicomanía. Entre 1933 y 1948 funcionó dentro de los terrenos de La Castañeda, pero operando de manera autónoma, el Hospital Federal de Toxicómanos, creado en el marco del Reglamento Federal de Toxicomanía promulgado el 27 de octubre de 1931. En dicho reglamento se establecieron "los procedimientos curativos a que quedarían sujetos los Toxicómanos", término utilizado para referirse a los consumidores de heroína, marihuana, cocaína y morfina ${ }^{6}$. Según el Reglamento, todas las instancias de Estado tenían la obligación de enviar al Hospital a los consumidores de marihuana, heroína, opio y cocaína, considerados bajo la doble calificación de transgresores y enfermos. El 3.9\% de los pacientes que ingresaron al Manicomio fueron diagnosticados con alguna forma de toxicomanía, es decir, una cantidad estimada de 2.397; mientras que al Hospital Federal de Toxicómanos fueron remitidos 1.625 entre 1933 y 1948, cuando fue clausurado.

En 1937 se encuentra el punto más alto de ingresos de toxicómanos (gráfica 3), y resulta interesante que $62.3 \%$ de los internados fueron remitidos por la Campaña contra el Alcoholismo y otras Toxicomanías. Esta información nos lleva a ampliar la mirada sobre la mencionada Campaña ya que en la historiografía ha sido expuesta la dimensión propagandística y pedagógica de ésta; sin embargo, estas fuentes nos hacen evidente la dimensión coercitiva de la misma. A las oficinas de la Campaña eran remitidos los toxicómanos quienes, a su vez, eran llevados al Hospital para ser «curados» en el marco de un proceso jurídico, ya que habían violado el Reglamento. Por su parte, en 1949 repuntó nuevamente la cantidad de ingresos de toxicómanos, y la característica principal de los que entraron en dicho año es que 63.3\% fueron remitidos por instituciones de seguridad o reclusión, como la Penitenciaria y la Procuraduría General de la República, además, el promedio de estancia fue inferior a un mes.

Las estancias de los pacientes consumidores de alguna de las mencionadas sustancias prohibidas eran bastante breves: 3.6 meses para quienes estaban en el Manicomio y 1.5 meses para los internos del Hospital de Toxicómanos. Menos tiempo de estancia y más reingresos son dos indicadores que caracterizan a esta población, donde la internación, al igual que en el caso del alcoholismo, tuvo una dimensión disciplinaria.

Según lo anterior, la reducción en el número de ingresos de los alcohólicos a partir de 1929 y el aumento en la cantidad de toxicómanos desde 1934 fueron procesos vinculados a las medidas tomadas por el Estado, por una parte con respecto a la acción pedagógica para reducir el consumo de bebidas alcohólicas, y por otra, a la penalización del consumo de las llamadas drogas «heroicas».

\section{DOS NOVEDADES: EL ELECTROENCEFALOGRAMA Y LA PENICILINA}

En cuanto a la epilepsia encontramos que, a partir de 1934, comenzó un incremento en la cantidad de pacientes con esta enfermedad. Entre 1910 y 1934 ingresó $35.24 \%$ del total de epilépticos, mientras que de 1934 a la clausura del Manicomio fueron confinados más de $64.75 \%$ (gráfica 4). Un aspecto a destacar es que si comparamos el motivo de alta por muerte antes y después de 1934, encontramos que en el segundo periodo se redujo considerablemente la mortalidad 
$18.86 \%$, frente a $39.1 \%$ de 1910 a 1933 , hecho que se podría atribuir a las nuevas terapias "eléctricas», intensificación de regímenes alimenticios e introducción de nuevos fármacos anticonvulsivos. Sin embargo, otros datos nos llevan a cuestionar la supuesta eficacia, ya que, aunque se redujo la mortalidad, el tiempo de estancia iba en aumento. Tan solo en la década de 1930 los pacientes epilépticos llegaron a permanecer en la institución cuatro años en promedio (48.73 meses), mientras que en las décadas anteriores permanecieron poco más de 2 años y medio (31.1 meses). El aumento de diagnósticos con epilepsia aunado al incremento del tiempo de estancia generó problemas de sobrecupo y falta de camas en los pabellones correspondientes. ${ }^{7}$ Ahora bien, ¿qué razones institucionales explican este incremento de la población psiquiátrica general y con epilepsia en particular?

Los diagnósticos de epilepsia se concentraron en cuatro grupos: epilepsias esenciales, constitucionales, jacksoniana y focales (tabla en Anexo). Entre 1910 y 1932, la epilepsia esencial o idiopática establecida por Jean Étienne Esquirol en 1838, fue la más diagnosticada debido a su fácil reconocimiento. Podía deberse a causas externas (contusiones), internas (una lesión cerebral) y morales (por ejemplo, el miedo intenso). A partir de 1933, se observa que la epilepsia esencial fue paulatinamente desplazada por los diagnósticos de epilepsias orgánicas y constitucionales, caracterizadas por una lesión específica o focal en los centros nerviosos causantes de las crisis convulsivas. Ahora bien, este desplazamiento conceptual respondió a dos factores de importancia diagnóstica y clínica: la introducción del electroencefalograma en los inicios de 1940 y la influencia directa de la escuela neurológica de Boston, encabezada por Gibbs, Davis y Lennox, que dieron gran impulso al EEG para el estudio clínico de la epilepsia (Postel y Quétel, 1987).

En este sentido, podemos afirmar que el incremento de la población con diagnóstico de epilepsia durante este periodo también respondió a la introducción de nuevos instrumentos de detección y a la asimilación teórica de un conjunto de conocimientos específicos acerca del funcionamiento cerebral. Lo anterior nos remite a una relación entre la implementación tecnológica y el diagnóstico de la epilepsia. A partir del electroencefalógrafo comenzó a «verse» dicha patología, permitiendo que pacientes que en otro momento habrían recibido un diagnóstico diferente (como dementes o retrasados mentales, por ejemplo), fueran ahora considerados epilépticos.

Otra afección, cuyo diagnóstico se redujo como resultado de una innovación tecnológica, fue la parálisis general progresiva (PGP), o sífilis terciaria. Esta afección preocupó a los médicos de La Castañeda por tratarse de un serio problema de salud pública con un tratamiento muy incierto ${ }^{8}$. Si bien en 1927 inició en el país la más importante "batalla» para contener

Gráfica 4. Ingresos por año de pacientes con Epilepsia y Parálisis General Progresiva al Manicomio La Castañeda, 1910-1968

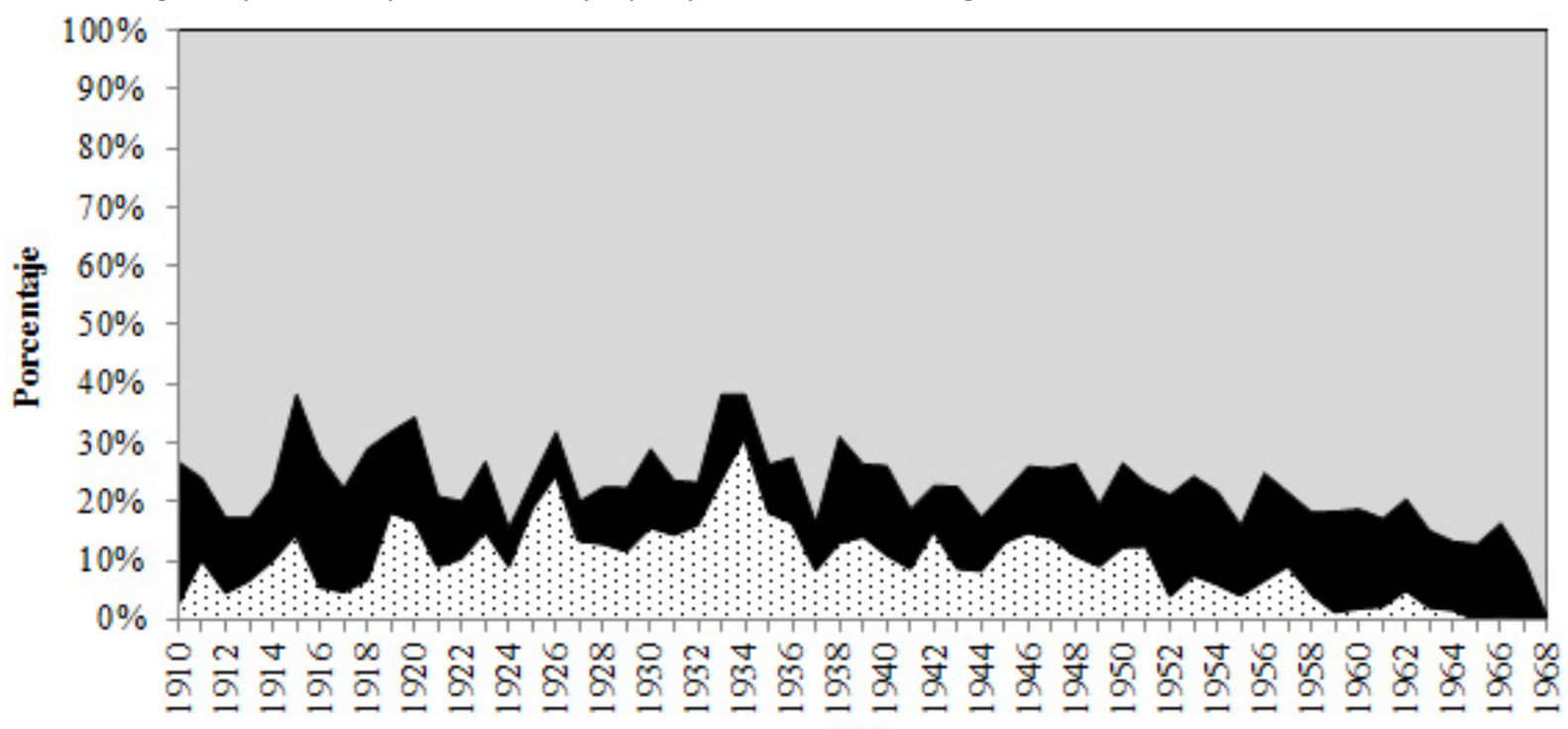

Año

\section{SÍFILIS $\quad$ EPILEPSIA $\square$ POBLACIÓN GENERAL}


la sífilis mediante la Campaña Nacional Antivenérea (Gudiño, 2009, pp. 133-161; Bliss, 1999) los ingresos de pacientes con dicha afección en La Castañeda permanecieron relativamente estables hasta 1934, cuando se alcanzó el mayor número. Una posible hipótesis nos llevaría a la etiología de la enfermedad, ya que sólo en el estado terciario de su evolución se presentan los cuadros de demencia, momento en que el treponema sifilítico invade el cerebro del infectado. Como esto ocurre 10 o 15 años después de contraer la enfermedad, podemos suponer que gran parte de los pacientes se infectaron en México durante la contienda bélica en el marco de la Revolución (1910-1920), años donde se perdieron los controles de la autoridad sanitaria sobre prostitutas y militares, la población más diagnosticada en el Manicomio con esta afección.

Por el contrario, a partir de 1950 observamos un decrecimiento constante en los ingresos de pacientes con parálisis general progresiva, posiblemente por la introducción de la penicilina en los dispensarios antivenéreos. El antibiótico controlaba la afección antes de que llegase al cerebro, impactando en la reducción de ingresos. En relación con el uso del antibiótico en La Castañeda, el 19 de diciembre de 1944 fue aprobado por la Junta del Consejo Psiquiátrico dirigida por Edmundo Buentello, el tratamiento a los pacientes neuroluéticos que reunieran ciertos requisitos por medio de la penicilina, particularmente entre aquellos que la malarioterapia o la electro- pirexia no había resultado útil ${ }^{9}$. Además, se estipuló que quienes estaban en calidad de «indigentes» recibirían las inyecciones de manera gratuita, a diferencia de los "pensionistas", quienes debían pagar por el servicio. Un año después, y gracias a la gestión del entonces director, Leopoldo Salazar Viniegra, la penicilina quedó integrada al cuadro básico de medicamentos de La Castañeda ${ }^{10}$. Así, tanto en el Manicomio como en los dispensarios antivenéreos se suministró penicilina, de manera tal que hubo una reducción en la cantidad de pacientes que ingresaron con parálisis general progresiva después de 1950.

\section{LA RECEPCIÓN DE KRAEPELIN Y LA CLÍNICA PSIQUIÁTRICA}

La esquizofrenia ha sido considerada la enfermedad mental por excelencia del siglo XX, lo cual le ha conferido un espacio privilegiado en la historiografía. En diversas instituciones psiquiátricas este diagnóstico fue el más numeroso (Garrabé, 1996; Novella y Huertas, 2010; Golcman, 2015), hecho que ocurre también en La Castañeda donde fue la enfermedad mental más diagnosticada: $19.9 \%$ de la población total presentó algún tipo de esquizofrenia. Si observamos la gráfica 5 encontraremos tres momentos en los que se evidencia un aumento en el porcentaje de esquizofrénicos ingresados: entre 1910 y 1925, cuando se diagnosticaba como demencia precoz, afectó al $10 \%$ de los internos, porcentaje que ascendió a $15.6 \%$ entre 1926 y 1954, y alcanzó un máximo entre 1955 y 1968 llegan-

Gráfica 5. Ingresos por año de pacientes con Psicosis Maniaco Depresiva, Paranoia y Esquizofrenia, 1910-1968

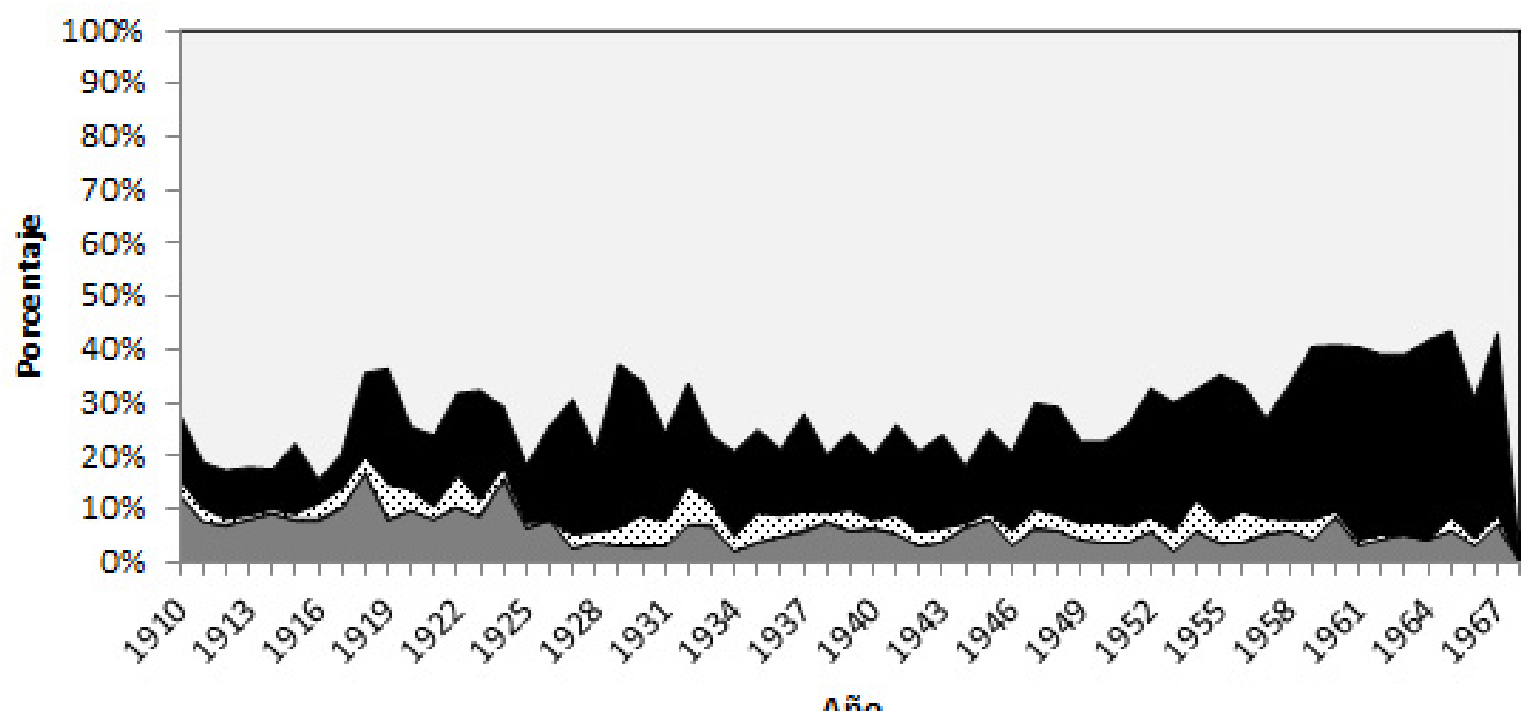

PSICOSIS MAN IACO DEPRESIVA QPPARANOIA aESQUIZOFRENIA DPOBLACIÓNGENERAL 
do a $22.7 \%$. Es más, en la gráfica 5 se puede observar que entre 1954 y 1964 casi se duplicó el porcentaje de esquizofrénicos. Ahora bien, ¿cómo explicar este aumento cuando la población total del Manicomio estaba estancada en su crecimiento? Debido a las limitaciones propias de nuestra base de datos no es posible determinar si hubo algún cambio contextual que generó un aumento en la cantidad de pacientes que fueron diagnosticados como esquizofrénicos.

El mencionado aumento va aparejado con otro proceso interesante: si vemos el tiempo de estancia de los esquizofrénicos en una perspectiva diacrónica encontramos que poco a poco se fue reduciendo: entre 1910 y 1925 fue de 49.1 meses, muy por encima del promedio general (18 meses); entre 1926 y 1953 se redujo a 33.1 meses; y entre 1954 y 1968 fue de solo 11.6 meses. Así, estamos frente a un incremento en la cantidad de pacientes diagnosticados con esquizofrenia, y una simultánea reducción en el tiempo de internamiento; rasgo paradójico ya que en otras instituciones psiquiátricas del siglo XX tanto en Europa como en las Américas, la población psicótica tendía a estancias prolongadas y fallecía después de muchos años de encierro. Los mencionados cortes cronológicos tienen un correlato: coinciden con dos cambios que tuvieron lugar en el campo de la clínica de la psicosis, más exactamente, con la recepción de Kraepelin y con la introducción de terapias farmacológicas ${ }^{11}$.

Desde finales del siglo XIX y hasta inicios de la década de 1920, los médicos mexicanos presentaron una clara preferencia por la medicina francesa, y el terreno psiquiátrico no fue la excepción ya que los pocos textos que se publicaron evidencian un conocimiento de autores como Phillipe Pinel y Jean Étienne Esquirol, pasando por Jules Falret, François Baillarger, August Morel, hasta Emmanuel Regis, Jean Marie Charcot, Ernest Dupré, Pierre Janet, entre otros (Morales, 2008). Dos manifestaciones tardías de esta preferencia por los autores franceses son las tesis Breves apuntes sobre las alucinaciones (1917), la cual se fundamenta en Janet y en Tanzi, y Estudios sobre la confusión mental (1920) donde se toma como referente a Chaslin, Seglas y Regis ${ }^{12}$.

La adopción del modelo de Kraepelin a partir de 1925 provocó un crecimiento en la población diagnosticada con esquizofrenia y de manera simultánea, se presentaron tesis en la Escuela de Medicina que dan cuenta de la recepción de este psiquiatra: Estudio clínico de la esquizofrenia y de sus diferentes formas, presentada por Guillermo Dávila en $1925^{13}$ y Psicosis maniaco-depresiva (Kraepelin) presentada por Roberto Portilla Velázquez en $1927^{14}$. La primera generación de psiquiatras mexicanos nació con el cambio de siglo, en la década de 1920 fueron estudiantes y en la siguiente década formaron parte del personal de La Castañeda. Entre los que se interesaron en la clínica de la psicosis se encuentran Samuel Ramírez Moreno, Edmundo Buentello, Leopoldo Salazar Viniegra y Manuel Guevara Oropesa (Ríos, 2016). Los tres primeros publicaron artículos donde daban muestra de la adherencia a las propuestas de Kraepelin, mientras que Guevara Oropesa pese a no publicar trabajos al respecto, se dedicó a la enseñanza de la clínica en La Castañeda. ${ }^{15}$

La recepción del modelo kraepeliniano se puede observar también en los pacientes con retraso mental, pues los términos clásicos franceses (idiocia, imbecilidad y debilidad mental) fueron sustituidos por el diagnóstico de «oligofrenia». De 1910 hasta 1928 los principales diagnósticos de retraso mental fueron los propuestos por Alfred Binet y Théodore Simon en $1907^{16}$ : idiocia (15\%), imbecilidad (60\%) y debilidad mental (25\%). Dicha clasificación, que seguía el paradigma degeneracionista, entendía el retraso como una "anormalidad» o un signo de degeneración que se evidenciaba por las malformaciones físicas y la diferencia entre la edad mental y la edad física. Sin embargo, en 1931, cuando Manuel Guevara Oropesa inició un proyecto de reorganización de los pabellones de La Castañeda, se adoptó el término oligofrenia y, en consecuencia, se inauguró el Pabellón de Oligofrénicos ${ }^{17}$ de hombres y mujeres destinado a los pacientes con retraso mental. A partir de esta fecha el diagnóstico de oligofrenia creció hasta el punto de que en 1942 el $100 \%$ de los pacientes ingresados por retraso fueron etiquetados con este diagnóstico y en 1953 el $90 \%$. Este aumento en el porcentaje de oligofrénicos implicó, además, que el retraso mental fuera concebido por los psiquiatras como un daño neurológico que afectaba a todos los órganos y que detenía el desarrollo psíquico del afectado. Para ello se establecieron nuevos parámetros de diagnóstico que iban acordes al modelo kraepeliniano: los diagnósticos debían iniciar con la palabra "oligofrenia», seguida por los factores causales (traumas, infecciones, entre otras) $y$, finalmente, por el signo o consecuencia orgánica del daño (malformaciones, estigmas degenerativos o grado de retraso psíquico $)^{18}$.

¿En qué consistió la propuesta tan aceptada de Kraepelin para la comprensión de las psicosis endógenas? Como es bien sabido, desde la medicina hipocrática se reflexionó sobre la etiología y sintomatología de las diferentes formas de locura. Sin embargo, a fines del siglo XIX hubo una radical transformación 
en el discurso psiquiátrico, hecho que tuvo una repercusión directa en la forma de concebir y clasificar las enfermedades mentales a lo largo del siglo XX. Los historiadores que han analizado este cambio de paradigma han señalado que fue el final de la tradición del «alienismo» francés como vanguardia, y el inicio del modelo de "las enfermedades mentales», donde Kraepelin se convertía en el principal exponente de una clínica psiquiátrica moderna (Huertas, 2005; Lantéri-Laura, 2000; Álvarez, 2008). Dicho cambio tuvo tres características. En primer lugar, se eliminó la idea de locura como entidad única con diferentes manifestaciones clínicas: manía, melancolía, demencia, idiotismo. En su lugar, el nuevo modelo impuso la idea de la existencia de diversas enfermedades mentales, cada una con sus especificidades tanto en la etiología, como en los síntomas y en la evolución. En segundo lugar, para comprender cada una de las enfermedades mentales, se introdujo la dimensión diacrónica, es decir, era obligatorio conocer los antecedentes, los primeros síntomas (según los acompañantes del paciente), los que se presentaban al ingresar a la institución psiquiátrica y la evolución de los mismos. Ello impedía diagnosticar a partir de la observación de la situación del paciente en un momento particular, ya que era necesario reconstruir la historia de vida, la evolución de los síntomas y sólo así determinar el diagnóstico. Es decir, incluyó la variable diacrónica ya que sólo al incorporar la perspectiva histórica en la clínica, era posible emitir un diagnóstico certero (Hoff, 2012, p. 309). A partir de allí emergía la gran diferencia: los que se recuperaban y los crónicos sin remedio. Y la tercera característica fue la articulación de los síntomas motrices con los cognitivos.

En la conceptualización de la psicosis a fines del siglo XIX fueron fundamentales los trabajos de Karl Kahlbaum, Alois Alzheimer, Eugen Bleuler, Ewald Hecker y Emil Kraepelin. De manera particular, la amplísima difusión del Tratado de Kraepelin, reeditado 12 veces entre 1895 y 1915 permitió que sus propuestas tuvieran un gran impacto. Kraepelin, en la sexta edición de su Tratado, planteó la dicotomía de las psicosis endógenas, al diferenciar primero la demencia precoz, marcada por la cronicidad, y la psicosis maniaco-depresiva con un buen o mejor pronóstico. Kraepelin afirmó:

lo que me convenció de la superioridad del método clínico de diagnóstico (adoptado en este caso) sobre el método tradicional, fue la seguridad con la que se podía predecir (en conjunción con nuestro nuevo concepto de enfermedad) el curso que tomarían los sucesos (Hoff, 2012, p. 333).
Para Kraepelin, la demencia precoz se definía por un debilitamiento de las actividades emocionales que constituyen el motor de la volición, y una pérdida de la unidad interna, ya que las emociones no corresponden con las ideas. La definió como «una serie de estados, cuya característica común es la destrucción peculiar de las conexiones internas de la personalidad psíquica» (Kraepelin, 2008, p. 27). Por consiguiente, la demencia precoz no estaría definida por un conjunto de síntomas sino por «conexiones internas» entre los mismos.

Gracias a Eugene Bleuler, el término demencia precoz fue remplazado por esquizofrenia, fenómeno que tuvo lugar en La Castañeda entre 1926 y 1930, años que coinciden con el ya mencionado cambio generacional ${ }^{19}$. Según German Berrios, la esquizofrenia se convirtió en una entidad de tal amplitud que le otorgó nombre a entidades que otrora eran denominadas de diferentes formas. Es como una especie de meta-concepto bajo el cual se agruparon diversos diagnósticos definidos desde la tradición psiquiátrica francesa. Así, el aumento de los diagnósticos de esquizofrenia puede ser considerado como una especie de colonización de un concepto en el lenguaje y en la práctica clínica (Berrios, et al., 2003). Por lo anterior, el progresivo aumento de la esquizofrenia puede ser entendido como un cambio en la mirada clínica que en un principio recurría a la psiquiatría francesa pero fue progresivamente desplazada por el modelo kraepeliniano.

Por otra parte, la notable reducción en el periodo de internación de los esquizofrénicos podría explicarse a partir del uso masivo de los antipsicóticos, particularmente de la clorpromazina (largactil) y la perfelacina (trilafón) desde $1952^{20}$. El problema radica en que no hubo un aumento en la cantidad de pacientes que salieron en remisión, es decir, que pese a las novedades terapéuticas, la mayoría de los esquizofrénicos, como el resto de la población de La Castañeda, era dada de alta por solicitud de la familia. Por consiguiente, otra hipótesis es que la reducción en el periodo de encierro puede ser el resultado de la labor realizada por la Sección de Trabajo Social en La Castañeda, espacio desde el cual se buscaba vincular a las familias en el cuidado de los pacientes psiquiátricos ${ }^{21}$. Las trabajadoras sociales tenían la tarea de visitar a las familias de los pacientes, explicarles la necesidad de que se hicieran cargo de sus parientes locos, y enseñarles a suministrar medicamentos y ofrecerles una buena calidad de vida. No obstante, sólo investigaciones posteriores a partir de los expedientes clínicos nos permitirán calibrar la eficacia tanto de los tratamientos farmacológicos como de la Sección de Trabajo Social de La Castañeda. 


\section{CONCLUSIONES}

Este texto ha sido un primer intento por abordar de manera global las características principales de los pacientes de La Castañeda. Como rasgo general encontramos que el paciente promedio tenía 33.6 años de edad y pasó encerrado 18.1 meses antes de que fuese dado de alta por solicitud de la familia o por no haber regresado de permiso. Además, la alta proporción de pacientes que fueron dados de alta por solicitud de la familia nos pone de manifiesto el determinante papel de las redes parentales a la hora de asumir el cuidado de los pacientes, lo cual significa que, desde la perspectiva cuantitativa, el encierro prolongado de sujetos en estado de cronicidad no fue un problema que aquejara al Manicomio General.

En segundo lugar, el análisis según los diferentes diagnósticos pone de relieve que la población psiquiátrica de La Castañeda, lejos de ser homogénea, fue diversa y plural, razón por la cual no es posible definir una periodización que dé cuenta de los cambios ocurridos en todos los pacientes. Por el contrario, la reducción en la cantidad de alcohólicos y el aumento de toxicómanos obedeció a factores externos, a saber, políticas públicas encaminadas al control de estas «patologías sociales», hábitos asumidos por el Estado como una amenaza para el proyecto de nación. Por su parte, el aumento en el diagnóstico de la epilepsia en 1934 y la reducción en la cantidad de pacientes

\section{NOTAS}

1 Investigación realizada con el apoyo económico del Programa UNAM-DGAPA-PAPIIT, IN 400313. La base de datos utilizada para este artículo fue elaborada por Daniel Vicencio, José Antonio Maya, Alejandro Giraldo, Alejandro Salazar, Alicia Sandoval, Anahí Toledo y Sandra Ayala, bajo la coordinación de Ximena López Carrillo. Los autores agradecen los comentarios recibidos por los dictaminadores.

2 Enrique Aragón (1943). "La enajenación mental en México", Mis 31 años de académico, México, Academia Nacional de Medicina de México, 1, pp. 415-470.

3 Dirección General de Bioestadística (1964). Primera Investigación Nacional de Enfermos Neurológicos y Psiquiátricos: (periodo del 20 al 30 de junio de 1960) México, Talleres Gráficos de la Nación.

4 Archivo Histórico de la Secretaría de Salud (AHSS), Fondo Manicomio General (F-MG), Sección Expedientes Clínicos (S-EC), Caja 21, Exp. 1344.

5 “Un notable alienista alemán en México. Viene al país con el propósito de hacer investigaciones científicas", El Universal, domingo 10 de mayo de 1925, p. 1-11. ingresados con parálisis general progresiva después de 1950 se explica a partir de importantes avances tecnológicos: el electroencefalograma y la penicilina. El primero permitió ubicar lesiones cerebrales en pacientes que sin tal aparato probablemente hubieran sido diagnosticados de diferentes formas, mientras que el uso masivo del antibiótico en los dispensarios antivenéreos redujo notablemente la cantidad de pacientes con sífilis terciaria.

Finalmente, los diagnósticos nos permiten ver la temprana recepción de Emil Kraepelin en La Castañeda: la esquizofrenia en sus tres formas (simple, hebefrénica y paranoide) y la psicosis maniaco-depresiva son muestra de ello; además, las publicaciones sobre dichas afecciones nos permiten corroborar la influencia de este psiquiatra en el ejercicio de la clínica. Sin embargo, pese a la cronicidad y las prolongadas estancias que se esperan de los pacientes con esquizofrenia, estas últimas fueron breves y concluyeron, en su mayoría, con el alta solicitada por la familia o por no regresar del permiso. Este hecho nos regresa a un tema que ha sido central en la historiografía de la psiquiatría: el lugar que ocupa la familia como instancia en interacción constante con el manicomio; en el caso mexicano, en lugar de abandonar a sus parientes en manos de una institución estatal, hubo una notable tendencia en las familias a solicitar el alta y hacerse cargo de sus propios locos.

6 El Reglamento Federal de Toxicomanías se publicó en 1936 en la revista Manicomio, (3-4), pp. 21-31.

7 Para 1936 y a petición de la Beneficencia Pública, el Departamento de Estadística del Manicomio General informó que el pabellón de Epilépticas contaba con seis salas en las que había "46-42-39-39-19-16 número de pacientes". Enfatizaron que hacían falta quince camas para cubrir la demanda. En el Pabellón Epilépticos existían cuatro salas, con "30-4728-45" número de camas, con "un faltante de 30 camas". Los encargados de los respectivos pabellones lamentaron la falta de espacio, en caso de cubrir la demanda de camas, "no había lugar para ponerlas". AHSS, F-MG, Sección Administrativa (Sec-A), Caja 22, Exp. 11.

8 Samuel Ramírez Moreno (1924), "Parálisis general progresiva: estudio acerca de la parálisis general progresiva", Universidad Nacional de México, Facultad de Medicina, tesis de licenciatura en Médico Cirujano, (Samuel Ramírez Moreno, 1928) "Tratamiento de la parálisis general progresiva", Gaceta Médica de México, 19(5), pp. 252-280, 331-367; (Samuel Ramírez Moreno, 1937) "Valoración a través del tiempo de la terapéutica en la parálisis general progresiva", Gaceta Médica de México, 67(3), p. 228-250 
9 AHSS, F-MG, Sec-A, Caja 46, Expediente. 2.

10 AHSS, F-MG, Sec-A, Caja 46, Expediente 2.

11 AHSS, F-MG, Sec-A, Caja 31, Expediente 1.

12 Gilberto de la Garza (1917), “Breves apuntes sobre alucinaciones, algunas de las principales teorías que explican su mecanismo", Universidad Nacional de México, Facultad de Medicina, tesis de licenciatura en Médico Cirujano; Julio Rodríguez Caballero (1920), "Estudio sobre la confusión mental", Universidad Nacional de México, Facultad de Medicina, tesis de licenciatura en Médico Cirujano.

13 Guillermo Dávila (1925). "Estudio clínico de la esquizofrenia y de sus diferentes formas", Universidad Nacional de México, Facultad de Medicina, tesis de licenciatura en Médico Cirujano.

14 Roberto Portilla Velázquez (1927), "Psicosis maniaco-depresiva (Kraepelin)", Universidad Nacional de México, Facultad de Medicina, tesis de licenciatura en Médico Cirujano.

15 Samuel Ramírez Moreno (1942), “Causas y tratamiento de la esquizofrenia”, Gaceta Médica de México, 74(1), 1944,

\section{BIBLIOGRAFÍA}

Ablard, Jonathan (2008), Madness in Buenos Aires: patients, psychiatrist and the argentine state, 1880-1983. Calgary, University Press

Agostoni, Claudia (2003), Monuments of Progress. Modernization and Public Health in Mexico City, 1876-1910. México, Universidad Nacional Autónoma de México, Instituto de Investigaciones Históricas, University of Calgary Press, University Press of Colorado.

Álvarez, José María (2008), La invención de las enfermedades mentales. Madrid, Gredos.

Berrios, Germán, et al. (2003), "Schizophrenia: a conceptual history", International Journal of Psychology and Psychological Therapy, 3 (2), pp.111-140.

Berrios, Germán y Roy Porter (eds.) (2012), Una historia de la psiquiatría clínica. Madrid, Triacastela.

Bliss, Katherine (1999), "The Science of Redemption: Syphilis, Sexual Promiscuity, and Reformism in Revolutionary Mexico City", The Hispanic American Historical Review, 79, pp. 1-40.

Carrillo Farga, Ana María (2010), Epidemias, saber médico y salud pública en el porfiriato, tesis de doctorado. México, Universidad Nacional Autónoma.

Conseglieri Gámez, Ana María (2014), El Manicomio Nacional de Leganés en la posguerra española (1939-1952): Aspectos organizativos y clínico asistenciales, tesis de doctorado. Madrid, Universidad Complutense. p. 93- 116. Leopoldo Salazar Viniegra, "Raíces míticas de la esquizofrenia", Archivos de Neurología y Psiquiatría de México, 4(5,6), pp. 235-244.

16 Alfred Binet y Théodore Simon (1907). Les enfant anormaux. Guide pour l'admission des enfants anormaux dans les clases de perfectionement. Paris, Colin.

17 AHSS, F-MG, Sec-A, Caja 8, Expediente 2.

18 Alicia Quiroz (1956). “Oligofrenia y agresividad”, Universidad Nacional Autónoma de México, Facultad de Psicología, tesis de licenciatura.

19 Un análisis sobre la primera tesis presentada en México sobre la esquizofrenia en 1925 es Zenia Yébenes, 2014, pp. 200-230.

20 Agustín Caso (1965), "Terapéutica farmacológica de la psicosis, 12 años de investigación de drogas antipsicóticas", en Gaceta Médica de México, 95(10), pp. 927-938.

21 Alfonso Quiroz Cuarón (1941), "Escuela de Trabajadores Sociales", Criminalia, 7(8), p. 461-470.

Dirección General de Bioestadística (1964). Primera Investigación Nacional de Enfermos Neurológicos y Psiquiátricos: (periodo del 20 al 30 de junio de 1960) México, Talleres Gráficos de la Nación.

Garrabé, Jean (1996), La noche oscura del ser. Una historia de la esquizofrenia. México, Fondo de Cultura Económica.

Golcman, Alejandra (2015), "El diagnóstico de la demencia precoz y la esquizofrenia en Argentina, 1920-1940", Trashumante. Revista Americana de Historia Social, 5, pp. 150-172.

Grob, Gerald (1983), Mental Illness and American Society, 18751940. Princeton, University Press.

Grob, Gerald (1994), The Mad Among Us. A History of the Care of America's Mentally III. New York, Free Press.

Gudiño Cejudo, María Rosa (2008), “Educación higiénica y consejos de salud para campesinos en El Sembrador y El Maestro Rural, 1929-1934", Agostoni, Claudia (coord.), Curar, sanar y educar. Enfermedad y sociedad en México, siglos XIX y XX. México, Universidad Nacional Autónoma de México, Instituto de Investigaciones Históricas, Benemérita Universidad Autónoma de Puebla, pp. 71-97.

Gudiño Cejudo, María Rosa (2009), Campañas de salud y educación higiénica en México, 1925-1960. Del papel a la pantalla grande,Tesis doctoral en Historia. El Colegio de México.

Hoff, P (2012), "Kraepelin”. En: Germán Berrios y Roy Porter (eds.), Una historia de la clínica psiquiátrica, España, Ed. Tricastela. 
Huertas García-Alejo, Rafael (2005), El siglo de la clínica. Para una teoría de práctica psiquiátrica. Madrid, Novalia.

Huertas García-Alejo, Rafael (2012), Historia cultural de la psiquiatría. Madrid, Catarata.

Kraepelin, Emil (2008), La demencia precoz, Buenos Aires, Editorial Polemos.

Lantéri-Laura, Georges (2000), Ensayo sobre los paradigmas de la psiquiatría moderna. Madrid, Triacastela.

Mancilla Villa, Marta Lilia (2001), La locura de la mujer durante el porfiriato. México, Círculo Psicoanalítico Mexicano.

Mendoza García, Ma. Eulalia y Graciela Tapia Colocia (2010), Situación demográfica de México, 1910-2010. México, Consejo Nacional de Población, pp.11-24, [en línea], disponible en: http://www.unfpa.org.mx/publicaciones/ cuadro_4.pdf, [consultado el 13/11/2014].

Micale, Marc (1985), "The Salpetriere in the age of Charcot: An Institutional Perspective on Medical History in the Late Nineteenth Century", Journal of Contemporary History, 20 (4), pp.703-731.

Mier y Terán, Martha (1991), "Dinámica de la población en México: 1895-1990. El gran cambio demográfico". DemoS, (4), pp. 4-5, [en línea], disponible en: http://www.ejournal.unam.mx/dms/no04/DMS00402.pdf, [consultado el $13 / 11 / 2014]$.

Morales, Francisco (2008), La apoteosis de la medicina del alma: establecimiento, discurso y praxis del tratamiento moral de la enajenación mental en la Ciudad de México, 18301910, Tesis de licenciatura en Historia. Universidad Nacional Autónoma de México.

Novella, Enric y Rafael Huertas (2010), "El Síndrome de Kraepelin-Bleuler-Schneider y la conciencia moderna: Una aproximación a la Historia de la Esquizofrenia", Clínica y Salud, 21 (3), pp. 205-209.

Pérez-Rincón (1995), Breve historia de la Psiquiatría en México. México, Instituto Mexicano de Psiquiatría.

Postel, Jacques y Claude Quétel (1987), Historia de la psiquiatría. México, Fondo de Cultura Económica.

Renvoize, Edward y Allan W. Beveridge (1989), “Mental illness and the late Victorians: a study of patients admitted to three asylums in York, 1880-1884", Psychological Medicine, 19 (1), pp 19-28.

Ríos Molina, Andrés (2004), "Locos letrados frente a la psiquiatría mexicana a inicios de siglo XX", Frenia. Revista de Historia de la Psiquiatría, 6 (2), pp. 17-35.

Ríos Molina, Andrés (2009a), La locura durante la Revolución Mexicana. Los primeros años del Manicomio General La Castañeda, 1910-1920. El Colegio de México.
Ríos Molina, Andrés (2009b), “Un mesías, ladrón y paranoico en el Manicomio La Castañeda. A propósito de la importancia historiográfica de los locos", Estudios de Historia Moderna y Contemporánea de México, 37, pp. 71-96.

Ríos Molina, Andrés (2009c), "El Manicomio General La Castañeda en México. Sitio de paso para una multitud errante", Nuevo Mundo Mundos Nuevos, Debates, [en línea], disponible en: https://nuevomundo.revues.org/50242, [consultado el 29/06/2015].

Ríos Molina, Andrés (2010), "Indigencia, migración y locura en el México posrevolucionario", Historia Mexicana, 59 (4), pp. 1295-1337.

Ríos Molina, Andrés (2011), "La psicosis del repatriado. De los campos agrícolas en Estados Unidos al Manicomio La Castañeda en la ciudad de México, 1920-1944", Mexican Studies/ Estudios Mexicanos, 27 (2), pp.361-384.

Ríos Molina, Andrés (2016), Cómo prevenir la locura. Psiquiatría e higiene mental en México. México, Siglo XXI Editores, Instituto de Investigaciones Históricas, Universidad Nacional Autónoma de México.

Rivera Garza, Cristina (2001a), "Dangerous minds: Changins psychiatric Views of the mentally ill in Porfirian. Mexico, 1876-1911", Journal of the History of Medicine and Allied Sciences, 56 (1), pp. 36-67.

Rivera Garza, Cristina (2001b), "She neither respected nor Obeyed Anyone: Inmates an Psychiatrist Debate Gender and Class at the General Insane Asylum La Castañeda, Mexico, 1910-1930", Hispanic American Historical Review, 81, pp. 653-688.

Rivera Garza, Cristina (2001c), “Por la salud mental de la nación: vida cotidiana y Estado en el Manicomio General de La Castañeda, Mexico, 1910-1930", Secuencia. Revista de Historia y Ciencias Sociales, 51, pp. 57-89.

Rodríguez Kuri, Ariel (2010), Historia del desasosiego. La revolución en la ciudad de México, 1911-1922. El Colegio de México.

Sacristán, Cristina (2001), "Una valoración sobre el fracaso del Manicomio La Castañeda como institución terapéutica, 1910-1944", Secuencia: Revista de Historia y Ciencias Sociales, 51, pp. 91-120.

Sacristán, Cristina (2002), “Entre curar y contener. La psiquiatría mexicana ante el desamparo jurídico, 1870-1944", Frenia. Revista de Historia de la Psiquiatría, 2 (2), pp. 61-80.

Sacristán, Cristina (2003), "Reformando la asistencia psiquiátrica en México. La granja de San Pedro del Monte: los primeros años de una institución modelo, 1945-1948", Salud Mental. Revista del Instituto Nacional de Psiquiatría, 26 (3), pp. 57-65.

Sacristán, Cristina (2005a), “Por el bien de la economía nacional. Trabajo terapéutico y asistencia pública en el Manicomio de La Castañeda de la ciudad de México, 1929-1932", História, Ciências, Saúde-Manguinhos, 12 (3), pp. 675-692. 
Sacristán, Cristina (2005b), “'La locópolis de Mixcoac' en una encrucijada política: reforma psiquiátrica y opinión pública, 1929-1933". En: Sacristán, Cristina; Piccato, Pablo (coords.), Actores, espacios y debates en la historia de la esfera pública en la ciudad de México, México, Instituto de Investigaciones Dr. José María Luis Mora, Instituto de Investigaciones Históricas, pp. 199-232.

Sacristán, Cristina (2005c), "Historiografía de la locura y de la psiquiatría en México. De la hagiografía a la historia posmoderna", Frenia. Revista de Historia de la Psiquiatría, 5 (1), pp. 9-34.

Sacristán, Cristina (2008), “Para integrar a la nación. Terapéutica deportiva y artística en el Manicomio de La Castañeda en un momento de reconstrucción nacional, 1910-1940". En: Agostoni, Claudia (coord.), Curar sanar y educar. Enfermedad y sociedad en México, siglos XIX y XX, México, Universidad Nacional Autónoma, Instituto de Investigaciones Históricas, Benemérita Universidad Autónoma de Puebla, pp. 99-123.

Sacristán, Cristina (2010a), "La contribución de La Castañeda a la profesionalización de la psiquiatría Mexicana", Salud Mental, 33, pp. 473-480.
Sacristán, Cristina (2010b), "Ser o no ser modernos. La salud mental en manos del Estado Mexicano, 1861-1968", Espaço Plural, 10 (22), pp. 11-23.

Sacristán, Cristina (2013), "Los hospitales para dementes y el crecimiento de la ciudad". En: Salmerón, Alicia; Aguayo, Fernando, Instantáneas de la ciudad de México. Un álbum de 1883-1884, t. 2, México, Instituto Mora, Universidad Autónoma Metropolitana, Fomento Social Banamex, pp. 15-29.

Vázquez de la Torre, Paloma (2013), El manicomio nacional de Santa Isabel en Leganés durante la Guerra Civil Española (1936-1939): población manicomial y prácticas asistenciales, tesis de Doctorado. Universidad Complutense de Madrid.

Vicencio Muñoz, Daniel (2014), La Operación Castañeda. Una reforma asistencia de la psiquiatría en México, 1940-1968, tesis de maestría. Universidad Nacional Autónoma de México.

Yébenes, Zenia (2014), Los espíritus y sus mundos. Locura y subjetividad en el México moderno y contemporáneo. México, Gedisa, Universidad Autónoma Metropolitana. 


\section{ANEXO}

Tabla anexa. Clasificación de los diagnósticos de los pacientes de La Castañeda.

\begin{tabular}{|c|c|c|c|c|c|}
\hline & \multicolumn{3}{|c|}{ Diagnósticos } & Casos & (\%) \\
\hline $\begin{array}{l}\text { II. Alteraciones mentales en los } \\
\text { traumatismos cerebrales }\end{array}$ & \multicolumn{3}{|c|}{ Lesiones } & 7 & .1 \\
\hline $\begin{array}{l}\text { II. Alteraciones mentales en otros } \\
\text { procesos orgánicos cerebrales }\end{array}$ & \multicolumn{3}{|c|}{ Encefalitis, parkinson, corea, bradiquinesia, entre otros. } & 225 & 2.1 \\
\hline \multirow{16}{*}{$\begin{array}{l}\text { Alteraciones mentales en } \\
\text { intoxicaciones }\end{array}$} & \multirow{11}{*}{ Alcoholismo } & Alcoholismo & 138 & \multirow{11}{*}{2055} & 19.3 \\
\hline & & Alcoholismo agudo & 40 & & \\
\hline & & Alcoholismo cerebral & 88 & & \\
\hline & & Alcoholismo crónico & 497 & & \\
\hline & & Alucinosis alcohólica & 112 & & \\
\hline & & Confusión mental alcohólica & 88 & & \\
\hline & & Delirium tremens & 112 & & \\
\hline & & Dipsomanía & 28 & & \\
\hline & & Korsakoff & 36 & & \\
\hline & & Psicosis alcohólica & 874 & & \\
\hline & & Sin especificar & 42 & & \\
\hline & \multirow{5}{*}{ Toxicomanía } & Marihuana & 116 & \multirow{5}{*}{414} & 3.9 \\
\hline & & Heroína & 95 & & \\
\hline & & Morfina & 22 & & \\
\hline & & No definida & 174 & & \\
\hline & & Otras sustancias & 7 & & \\
\hline $\begin{array}{l}\text { IV. Alteraciones mentales en las } \\
\text { enfermedades infecciosas }\end{array}$ & \multicolumn{3}{|c|}{ Meningitis } & 18 & .2 \\
\hline \multirow{5}{*}{ V. Sífilis } & & mencia paralítica & 29 & \multirow{5}{*}{998} & \multirow{5}{*}{9.4} \\
\hline & & Neurolues & 163 & & \\
\hline & & PGP & 685 & & \\
\hline & & Sifilis & 88 & & \\
\hline & & Tabes & 33 & & \\
\hline \multirow{2}{*}{ VI. Senilidad } & \multicolumn{3}{|c|}{ Arterioesclerosis } & 4 & 0 \\
\hline & \multicolumn{3}{|c|}{ Senilidad y presenilidad } & 384 & 3.6 \\
\hline \multirow{4}{*}{ VII. Epilepsia } & & Epilepsia & 940 & \multirow{4}{*}{1369} & \multirow{4}{*}{12.9} \\
\hline & & oilepsia esencial & 345 & & \\
\hline & & epsia Jacksoniana & 25 & & \\
\hline & & Epilepsia focal & 59 & & \\
\hline
\end{tabular}




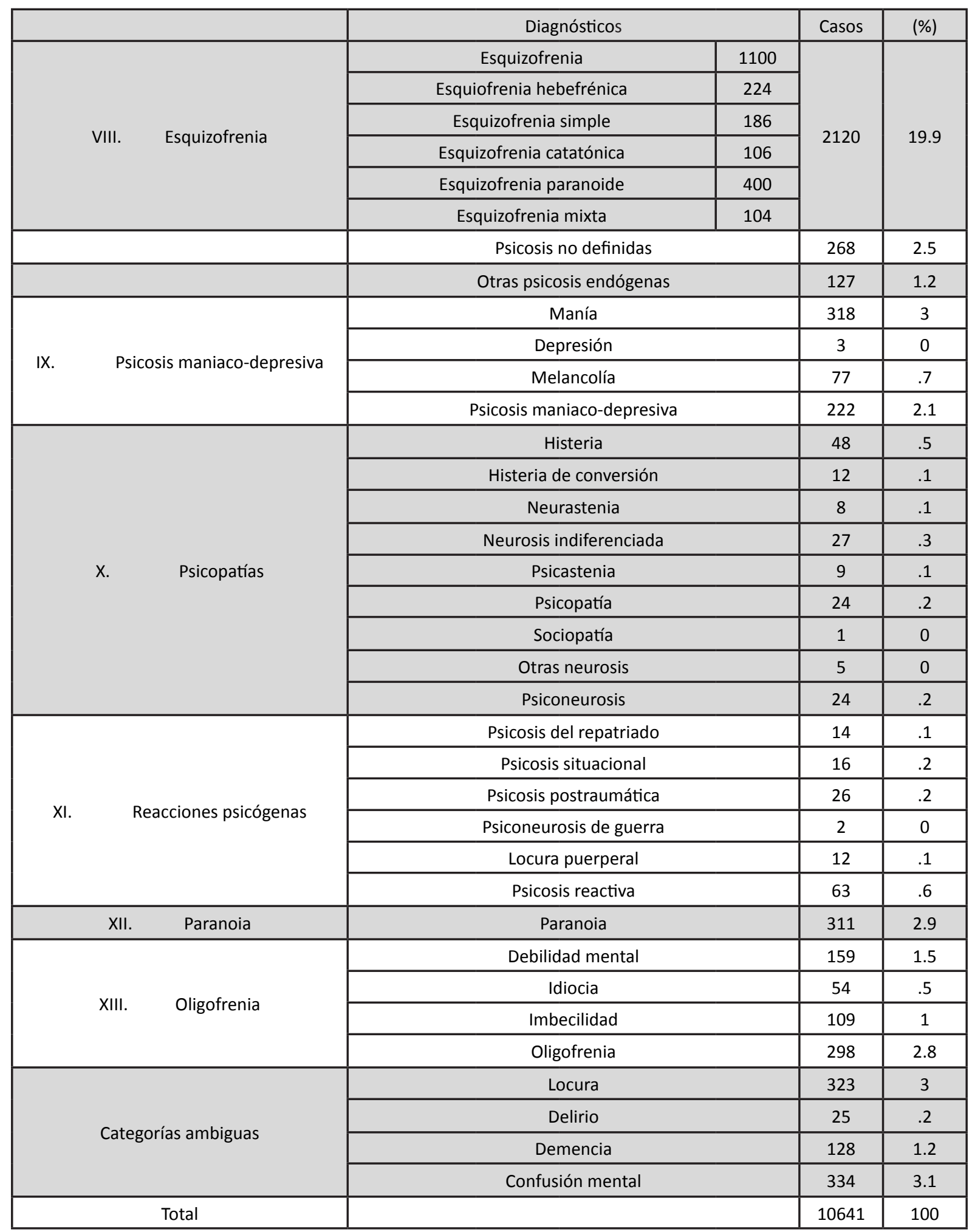

